\title{
A framework for the study of multiple realizations: the importance of levels of analysis
}

\author{
Morten Overgaard ${ }^{1,2 *}$ and Jesper Mogensen ${ }^{3}$ \\ 1 CNRU, Department of Psychology and Communication, Aalborg University, Aalborg, Denmark \\ 2 Cognitive Neuroscience Research Unit, Hammel Neurorehabilitation and Research Center, MindLab, Aarhus University, Aarhus, Denmark \\ 3 The Unit for Cognitive Neuroscience, Department of Psychology, University of Copenhagen, Copenhagen, Denmark
}

\section{Edited by:}

Mads Jensen, Aarhus University Hospital, Denmark

\section{Reviewed by:}

Alexander Maier, National Institute for Mental Health, USA

John Bickle, Mississippi State

University, USA

*Correspondence:

Morten Overgaard, Cognitive Neuroscience Research Unit, Hammel Neurorehabilitation and Research Center, MindLab, Aarhus University, Noerrebrogade 44, building 10G, DK-8000 Aarhus, Denmark. e-mail:mortover@rm.dk
The brain may undergo functional reorganizations. Selective loss of sensory input or training within a restricted part of a modality cause "shifts" within for instance somatotopic or tonotopic maps. Cross-modal plasticity occurs when input within a modality is absent - e.g., in the congenitally blind. Reorganizations are also found in functional recovery after brain injury. Focusing on such reorganizations, it may be studied whether a cognitive or conscious process can exclusively be mediated by one neural substrate - or may be associated with multiple neural representations. This is typically known as the problem of multiple realization - an essentially empirical issue with wide theoretical implications. This issue may appear to have a simple solution. When, for instance, the symptoms associated with brain injury disappear and the recovery is associated with increased activities within spared regions of the brain, it is tempting to conclude that the processes originally associated with the injured part of the brain are now mediated by an alternative neural substrate. Such a conclusion is, however, not a simple matter. Without a more thorough analysis, it cannot be concluded that a functional recovery of for instance language or attention is necessarily associated with a novel representation of the processes lost to injury. Alternatively, for instance, the recovery may reflect that apparently similar surface phenomena are obtained via dissimilar cognitive mechanisms. In this paper we propose a theoretical framework, which we believe can guide the design and interpretations of studies of post-traumatic recovery. It is essential to distinguish between a number of levels of analysis - including a differentiation between the surface phenomena and the underlying information processing - when addressing, for instance, whether a pre-traumatic and posttraumatically recovered cognitive or conscious process are actually the same. We propose a (somewhat preliminary) system of levels of analysis, which can be applied to such studies.

Keywords: neural substrate, cognition, consciousness, plasticity, brain injury, reorganization, levels of analysis, neural correlate of consciousness

\section{INTRODUCTION}

One of the primary concerns in the attempt to isolate some neural correlate of consciousness (NCC) is the question whether conscious content relates to brain processes in a fixed 1:1 pattern, or whether the same content may be realized in different brain processes. The answer to this question will have important theoretical consequences: Should the latter view be correct, it would seem futile to reduce or identify conscious content with one specific brain process. Instead, such a finding would support theories that allow a "one-to-many" relationship.

The experimental literature is already rich with examples showing that individual connections in the brain are constantly being modified, largely dependent upon how they are used (e.g., KellerPeck et al., 2001; Lee et al., 2006, 2008). Decades ago, it was shown that if the cortical organization for a sensory system is deprived of input, it will become activated at a later time in response to other, usually adjacent inputs (e.g., Yang et al., 1994; Weiss et al., 2000; Karl et al., 2001). Such reorganization seems to be found with any chosen method of analysis, be it changes in individual cells, networks of cells, or entire "modules."
However well-established the phenomenon of brain plasticity may be, the question whether we should conceive of mind-brain relations as 1:1 or 1:X remains unanswered. We believe this lack of success is mainly due to the lack of a proper conceptual and methodological framework to approach the issue: It seems difficult to decide whether two occurrences of a mental state or a brain state are identical or not. This decision must, however, be made in order to answer the over-all question about the relationship between the two kinds of states.

This paper aims to take the first steps toward such a framework, outlining an approach to analyze the problem, and giving examples of the issues being considered.

\section{BACKGROUND}

There is a constantly growing number of studies demonstrating that when a particular sensory input is lacking (due to peripheral loss - but without injury to the brain) a series of short- and long-term reorganizations take place within various levels of the brain systems representing the affected modality. Such reorganizations occur, for instance, within the somatotopic representations 
of various body-parts (e.g., Yang et al., 1994; Weiss et al., 2000; Karl et al., 2001) and the tonotopic representations of the auditory system (e.g., Robertson and Irvine, 1989; Scheich, 1991; Irvine, 2007; Thai-Van et al., 2007).

Individuals, who lost one hand to amputation (thereby depriving the somatosensory cortex of the contralateral hemisphere of normal input to the region specialized for processing hand-related information), have been studied extensively (e.g., Yang et al., 1994; Weiss et al., 2000; Karl et al., 2001). In such persons, the region of the primary somatosensory cortex, which used to represent the now amputated hand, does not remain inactive. Instead, the neighboring somatosensory regions (representing the arm and face, respectively) encroach on the now "vacant" area in such a way that the "input-lacking" cortical hand area is eventually fully taken over by inputs from arm and face, respectively. Plastic reorganizations of the somatosensory cortical map do, however, not only occur under conditions of a lacking input from parts of the body. Even changed experiences in terms of intensive training restricted to part of the body may be associated with a relative shift within somatosensory representations (e.g., Merzenich and Jenkins, 1993; Elbert et al., 1995; Xerri et al., 1996; Münte et al., 2002).

Showing that these plastic processes within the somatosensory system are not unique, the tonotopic representations of the auditory system appear also to undergo plastic changes due to changes in input or experience. Partial loss of input (e.g., due to restricted cochlear lesions) deprives the tonotopic representation of input within a restricted spectrum of frequencies. Upon such a manipulation, the representations of the neighboring frequencies expand to fill the "vacant" region of for instance the auditory cortex (e.g., Robertson and Irvine, 1989; Scheich, 1991; Irvine, 2007; Thai-Van et al., 2007). Within the frequencies, which have - due to such mechanisms gained an increased neural representation, the quality of frequency discrimination appear to be enhanced (Thai-Van et al., 2007). Learning experiences specific to a restricted tonal representation are also able to induce an increased representation of the frequencies in question (e.g., Scheich, 1991; Recanzone et al., 1993; Irvine, 2007) - and this increased representation within the tonotopic representation seems to be correlated with the quality of performance of the auditory discrimination task (e.g., Recanzone et al., 1993).

While the above examples deal with plastic reorganizations within one modality, there are also examples demonstrating the even more radical phenomenon of cross-modal plasticity. For instance, such a phenomenon has been found in the context of attempts to provide the blind with a "visual prosthesis." The idea of such research is to provide a - presently crude but nevertheless potentially useful - representation of the visual world via a somatosensory input. Captured by a camera, the image of the environment is transformed into a crude - "low-resolution" - representation in the form of a matrix with "active" and "inactive" pixels. This matrix is presented to the blind subject in the form of somatosensory stimulation. The matrix is brought into contact with a part of the body surface and "active" pixels are designated by for instance vibration or mild electric stimulation (while "inactive" pixels lack such a stimulation). In a number of these experiments the inputs have been simple geometric patterns - for instance letters of various orientations (e.g., Bach-y-Rita et al., 1969, 1998; Kaczmarek et al., 1991; Ptito et al., 2005). It has been demonstrated by Ptito et al. (2005) that both blind and normally sighted (but blindfolded) individuals can learn to utilize this device in solving discrimination tasks in which they are required to determine the orientation of letters. While the ability to acquire the task was similar in both groups, the neural regions activated during task performance turned out to differ. In both groups somatosensory cortical regions were activated but in the blind group (in contrast to the sighted individuals) additional activations were found in cortical regions normally associated with the processing of visual information (Ptito et al., 2005). In contrast, the sighted but blindfolded subjects demonstrated a cortical activation pattern similar to what would be expected when performing an ordinary tactile discrimination task (Ptito et al., 2005). It may be especially relevant that in the blind subjects, successful task solution was associated with activation of cortical regions, which in sighted individuals participates in mediation of visual tasks in which the spatial orientation of figures is to be determined. It should be noted that Ptito et al. (2005) studied individuals who were "early blind."

The most commonly studied aspect of cross-modal plasticity in the blind is the involvement of visual cortical areas in the performance of Braille reading (e.g., Gizewski et al., 2003; Ptito et al., 2008). There have been some doubts regarding the specificity of the involvement of visual areas in tactile discrimination tasks related to Braille reading (Gizewski et al., 2003). However, in a study directly addressing this issue, Gizewski et al. (2003) found that in early blind subjects somatosensory activation of visual areas was exclusively related to the performance of tactile discrimination tasks (including Braille reading) - but not elicited by electrical stimulation of the hand used in the performance these tasks.

The main focus of studies such as those described above is the ability of the brain to reorganize in ways that allow a structure or substructure to become functionally engaged within a domain, which is more or less dissimilar to the type of information processing, originally managed by the circuitry in question. Regarding shifts of somatotopic or tonotopic maps, the plasticity-induced novelty concerns different aspects within the same sensory modality, while - in cross-modal plasticity - other cases even demonstrate a shift to processing sensory information of another modality than the one to which the brain region is supposedly originally "programmed."

Normally, such results are taken to indicate that a given brain structure is able to subserve multiple "functions" - at least within the restrictions indicated by various studies (cross-modal changes may, for instance, most easily or maybe exclusively be achieved in case of early deprivation of a sensory modality, e.g., Ptito et al., 2005).

When seen from another angle, the above-described examples can also be seen as indications that the same "function" can be mediated by multiple neural substrates. This may not commonly be the focus of such studies, but when, for instance, an area previously specialized in processing information regarding a hand is able also to mediate face-related information processing, this may also be framed in terms of a "relocation" of face-related information processing. More clear-cut cases of an apparent "relocation" of functional mediation are, however, to be found within the literature dealing with the neural substrate of post-traumatic functional recovery.

It must, however, be remembered that theoretically an apparent "relocalization" of functional mediation (as a consequence of either a modified input to an intact brain or regional brain injury) might 
be accompanied by a "rewiring" of the local circuitry within the novel substrate of functional mediation. If the neural plasticity mediating the "relocalization" not only changes the input/output properties of the novel structure mediating a function, but also rewires the internal networks of this brain region, it could be argued that the entire process includes the creation of an actual copy of the neural network mediating the function within its original location. If this can be the case, a 1:1 relationship between function and neural processing could be preserved while the site of the mediating circuitry has just shifted to a novel structure.

Thus, it is important to consider how likely it is to find a (re) establishment of the basic circuitry of brain regions in the adult brain. This issue is pursued in more detail by Mogensen (2011a,c). During maturation, neurons undergo a number of changes making them less similar to the developing neurons, which originally formed the local circuits of the brain (e.g., Fawcett et al., 1989; Chen et al., 1995; Goldberg et al., 2002). These restrictions may, however, be somewhat outweighed by the ongoing neurogenesis in the adult brain - a neurogenesis which is further potentiated when the brain is hit by injury (e.g., Magavi et al., 2000; Scharff et al., 2000; Arvidsson et al., 2002; Nakatomi et al., 2002; Chen et al., 2004). These newly formed neurons may in the injured brain even receive some support in reaching relevant brain regions - upon injury, mature astrocytes are able to transform themselves into radial glial cells similar to those guiding neural migration (and thereby the arrival of newly formed neurons in the relevant brain structures) during development (e.g., Rakic, 1971, 1985; Leavitt et al., 1999). There may, however, be at least one crucial factor preventing the uninjured or injured - adult brain from recreation of networks similar to those formed during development: from the final stages of the original ontogenic development (and thereby formation of the basic circuitry of the brain) a number of factors associated with glial cells and myelin (e.g., Berry, 1982; Schwab and Thoenen, 1985; Schäfer et al., 2008) appear to prevent restructuring and presumably recreation of such basic circuitry. Especially important may be the astrocyte-produced chondroitin sulfate proteoglycans (CSPGs). These substances play an important role in the termination of the developmentally "critical periods" and thereby "consolidate" the originally formed circuitry in its "final" form (e.g., Pizzorusso et al., 2002; Berardi et al., 2004; McGee et al., 2005; Del Rio and Soriano, 2007; Schäfer et al., 2008). And the presence of these CSPGs apparently blocks an adult recreation of traumatically lost networks (e.g., Del Rio and Soriano, 2007; Schäfer et al., 2008).

Maybe one of the cognitive domains, which have been most extensively studied with respect to the mechanisms of posttraumatic functional recovery, is language. Numerous studies have scrutinized (so far without reaching consensus) the neural substrate of post-traumatic functional recovery of patients suffering various types of aphasia due to either stroke or traumatic brain injury (TBI).

Most patients suffering post-traumatic aphasia regain at least a partial proficiency of language, and such a recovery process is accompanied by reorganizational processes within various parts of the brain (e.g., Marsh and Hillis, 2006). These reorganizational processes may be affected by the type of traumatic impact, itself. For instance, gliomas may be removed in either a one- or two-step surgical procedure. And compared to the more massive impact of the single surgical intervention, the two-step procedure leads to a different type of post-traumatic reorganization (e.g., Duffau et al., 2002). Numerous methods have been employed in demonstrating the post-recovery substrate of language processing in patients suffering aphasia - some of the most common methods being imaging techniques (e.g., Perani et al., 2003) and physiological methods such as event-related potentials (e.g., Laganaro et al., 2008).

Aphasias primarily result from injury to the left hemisphere and the contribution of ipsilateral (left hemisphere) mediation of the reacquired linguistic functions have been demonstrated by for instance Szaflarski et al. (2010), Perani et al. (2003), Specht et al. (2009), and Meinzer et al. (2008) - the latter study demonstrating treatment-induced reintegration of various perilesional areas. The most common question asked within this field is, however, whether the contralateral, right, hemisphere plays a significant role in mediating post-traumatic recovery of language. The earliest case in which it was presumed that post-traumatic recovery upon expressive aphasia (also known as non-fluent aphasia - a condition characterized by difficulty producing speech on the background of a relatively preserved comprehension of language) was mediated by mechanisms within the right hemisphere (in this case the mirror-structure of Broca's area), was published by Barlow (1877). Doubts about this case remain, and Finger et al. (2003) concluded that we still know too little to fully interpret cases such as this one. However, without going into the details of whether a case like Barlow's manifest a true case of "vicariation" (the phenomenon that brain areas with different functions can sometimes assume or "take over" the function of an injured brain region - e.g., Finger and Stein, 1982; Slavin et al., 1988) it is clear that numerous studies - utilizing a variety of methods - have found an apparent involvement of structures within the right hemisphere in mediation of post-traumatic recovery in aphasia (e.g., Thomas et al., 1997; Thulborn et al., 1999; Ansaldo et al., 2002; Ansaldo and Arguin, 2003; Perani et al., 2003; Baumgaertner et al., 2005; Specht et al., 2009). In some cases (e.g., Thomas et al., 1997) there are indications that the patterns of shift toward a right hemisphere mediation of linguistic functions differ between types of aphasia. Furthermore, changes in the direction of language being mediated by the right hemisphere are often accompanied by internal reorganizations within the left hemisphere (where linguistic mediation may shift to uninjured regions). These reorganizations may lead to a more bilateral representation of language - due to the concurrent shift of linguistic mediation within the injured left hemisphere and to the contralateral, right hemisphere (e.g., Thompson et al., 2010). In most instances, the recovery-associated shifts toward right hemisphere mediation of language occur without the rehabilitative training specifically aiming at such a shift (but rather working in a more general way toward a recovery of the lost linguistic abilities). There are, however, cases in which (partly successful) training of aphasic patients have aimed at achieving a higher degree of right hemisphere mediation of linguistic tasks (e.g., Crosson et al., 2009 - who used a manipulation task performed with the patient's left hand to initiate naming trials and thereby obtain an independent right hemisphere activation which presumably can ease an interhemispheric shift of linguistic task mediation). It can often correctly be questioned to what extend the changes in neural activity observed via for instance fMRI in a recovering or recovered aphasic patient are specifically related to the reacquisition of the 
language. A post-traumatic change in activity within a given structure may be the consequence of any trauma-related process - e.g., "disinhibition" due to lack of input from the injured brain region. Or other processes which are not directly related to the recovery of language. Certain cases are, however, highly informative in this respect. Meinzer et al. (2007), for instance, studied the linguistic recovery of a bilingual aphasic patient. In this case activation of parts of the superior temporal lobe of the right hemisphere was exclusively associated with use of the trained language while no such activation was observed associated with the untrained language.

To summarize, post-traumatic functional recovery upon brain injury - such as in the instances described above-may demonstrate that performance of the recovered task is associated with activity within brain regions, which were not pretraumatically involved in mediation of such tasks. Such results are normally taken to indicate that in one way or another a task mediation originally achieved by the injured part of the brain is now accomplished by neural mechanisms not previously involved in these functional domains.

In philosophy of mind, Block and Fodor (1972) used plastic properties of the brain as evidence for multiple realization and as an argument for functionalism. However, all findings mentioned above would be fully consistent with the proposal that whereas the post-traumatic, recovered function, e.g., the ability to speak, has superficial similarities with the pretraumatic function, the exact language structure has changed according to the change in neural substrate. What appear to be similar surface manifestations of a cognitive process may - when further scrutinized - turn out to be more dissimilar than originally assumed. For instance, a posttraumatically recovered task performance may have allowed the injured individual to regain a full proficiency (to the level of what is achieved with an uninjured brain) but this recovery is nevertheless achieved via cognitive mechanisms which upon further scrutiny turns out to be dissimilar to those of normal individuals (e.g., Mogensen et al., 2004). The need for a more thorough analysis of the surface phenomena observed during functional recovery after brain injury has been both methodologically and theoretically discussed elsewhere (e.g., Mogensen and Malá, 2009; Mogensen, 2011a,b,c; Wilms and Mogensen, in press).

At the same time, however, and as illustrated in the example of aphasia, it seems one may be able to argue for and against multiple realization, embracing the view that while the ability to speak, as such, is re-established in a different way in the brain, the exact same way of speaking, when analyzed in all details, is forever lost. That is: while viewed superficially the task performance of the patient demonstrates that general linguistic abilities are reacquired, but a more detailed analysis of the post-traumatic cognitive processes reveals that the post-traumatically established linguistic processes are dissimilar to those seen pretraumatically. This, in and of itself, reveals, at least, two unanswered questions underlying the debate: In which sense can two instances of a mental phenomenon be said to be the same, and with which methods could such an identity be established?

Although such questions appear directly from an analysis of empirical research, they have been debated for decades in philosophy of mind. Notably, Shapiro $(2004,2008)$ discusses difficulties in considering multiple realization an immediately testable empirical hypothesis. Shapiro (2008) asks the reader to consider three watches: Two analog ones and one digital. The two analog watches differ in timekeeping properties in that one runs fast and the other late. Nevertheless, the difference can be explained by one respect - a spring in one of the watches is longer than in the other. Shapiro argues that timekeeping is realized in the same way in these two watches although they have slightly different timekeeping properties. Timekeeping, nevertheless, is realized differently in the digital watch, he claims. If one of the analog watches had the exact identical timekeeping properties as the digital one, despite the difference in physical properties, it would be evidence for multiple realization. So, the example challenges evidence for multiple realization in the brain: Are the physical differences, relevant to realization, only relevant for that realized property? If so, it is not a case of "true multiple realization."

Bechtel and Mundale (1999) argue that one central issue is that psychological as well as neural properties can be described with different "granularities." They argue that psychological properties often are described at a very "coarse" level of granularity when deciding whether two mental states are identical or not, whereas neural properties are described at a much "finer" level. In order to make a comparison between two mental states, and thus conclude anything about whether some observation is a case of multiple realization, it must first be resolved at which level, mental states are properly described. In Table 1 we provide a tentative framework for levels of analysis relevant to this issue.

\section{LEVELS OF ANALYSIS IN COGNITIVE PROCESSES}

To begin with, mental states may be compared at different levels. While the term "mental states" in itself is difficult to delineate and define, the first part of the paper will look at mental states as discussed in cognitive science: Functions which may be described as states or processes inferred from behavior.

Looking at cognitive states, we will tentatively suggest at least such three levels of analysis where a mental state may be defined and compared to other states.

At the most general level, it is a relatively simple task to determine whether or not an entire domain is intact, lost or fully recovered. Here, the question is simply if there is visual perception, language comprehension, or any such over-all function present. A person may loose her ability to understand language following an injury to the brain, but recover this ability. The ability may be only partially recovered, understanding just some words, but at this general level it is the same function as before. At this level, the presence or

Table 1 | Levels of analysis in the relation between cognitive and neural events. The number of levels and their labeling are tentative yet serve to show the approach

\begin{tabular}{ll}
\hline Level of analysis & Description \\
\hline Cognitive domain & General, functional manifestation level, \\
& e.g., "visual perception" \\
or "language comprehension" & More specific function related to a domain, \\
Task- and & e.g., "visual form discrimination" \\
domain-specific function & Basic-level operations, may be \\
Basic functions & described in mathematical terms
\end{tabular}


absence of an impairment is typically established without reference to specific test procedures, but rather by a more direct (e.g., "general clinical") observation of the individual. For instance, it may be immediately evident that an aphasic patient is unable or severely impaired when attempting to communicate via language. This can be established without reference to particular tests while more detailed observation and testing is necessary to perform a further characterization of the affected subdomains within language, perception, or other post-traumatically affected domain. Similar arguments can be made regarding the demonstrations of recovery at this level.

With regards to task- and domain-specific functions, the situation becomes more complex. First of all, some may question the conceptual division between this level and the level of cognitive domains, arguing that this is nothing but a more elaborate description of the different domains. However, as a patient after brain injury may have very specific dysfunctions, lacking, e.g., the ability to recognize faces but have no other perceptual and/or memory problems, we believe the two levels can be separated at least as different levels of description or analysis. Generally speaking, it is conceptually and scientifically useful and important to keep the two levels distinct. For instance, two different visual tasks can only be conceived of as "visual" because they can be described at a "cognitive domain" level as well as a more specific task-level.

Basic functions are prima facie very different than the task-/ domain-specific functions, as they should be conceived of as discretely operating systems without any direct manifestation at an experiential or a behavioral level. We consider these basic functions of the present model to be either identical to or at least at the same level as the Elementary Functions defined within the reorganization of elementary functions (REF) model (Mogensen and Malá, 2009; Mogensen, 2011a,c). Such Elementary Functions are basic information processing units ( $t$ o be viewed as a kind of programming modules for a modular programming system of cognition). Elementary Functions are not specific to any domain or task, since an individual Elementary Function contributes to multiple higher order programs - each of which has a more task- and domain-specific application. The characterization of an Elementary Function may be more easily achieved in the language of mathematics than in the terminology of psychology.

The three levels of the presently proposed model are not to be seen as hierarchical in the sense that one is reducible to the other. However, the model suggests a conceptual hierarchy in the sense that for instance task-specific functions are categorized based on the cognitive domain (e.g., visual or auditory perception, logical reasoning etc). One may reasonably question whether the "granularity" of the neural processes involved should not be considered as well. For instance, one might question whether reorganization after brain injury will not lead to a structuring of the postlesional areas that is similar to the prelesional ones in such a way that the conception of multiple realization this way will be challenged. However, considering such examples of recovering language abilities after massive injuries to the left hemisphere, such a worry seems to lean on a rather "coarse grained" notion of the involved brain processes. In other words, we argue that such a worry may be applicable to some cases but is less relevant to a more general discussion of the possibility of multiple realization per se.
In the analysis of the neural substrate of post-traumatic recovery, it is more often than not impossible to tell at which "level" a neural substrate of postrehabilitation task mediation is a "replacement" of what has been lost. In other words: it is, for instance, unclear to what extent the same information processing ("function") is present in the right hemisphere when recovering aphasic patients show an increased focal activation within that hemisphere. This situation is often a result of the understandable focus on clinical applications and the potentials of a therapeutic method with respect to achieving a successful rehabilitative process. It can be argued that the essential information is that: (1) the patient is clinically improving, and (2) activation and/or establishment of particular (partly unknown) neural processes within a particular region of the brain is associated with this clinical success. Theoretically, however, such a pragmatic approach can be unsuccessful and potentially misleading. Clinically, reduction or elimination at the surface level of the post-traumatic symptoms is the crucial issue. Theoretically, however, it is of essential importance to establish not only whether a general level of functional recovery is seen, but also whether the mechanisms of such a recovery include re-establishment of the exact information processing lost to trauma. If the issue of such a re-establishment is not addressed, a more superficial examination of the post-traumatic rehabilitation of patients can potentially yield a false impression that lost information processing is reacquired.

In quite a number of cases, e.g., visuospatial neglect, the incidence of severe symptoms is dramatically high in the acute phase, yet most seem to recover (Corbetta et al., 2005). Perhaps less dramatically, this picture is found in many different dysfunctions where the pattern of a reduction of symptoms when moving from the acute to the postacute phase is very different in the acute and postacute phase. In the acute phase, patients suffering neglect may show the well-known symptoms of not eating food on the left side of their plate, bumping into objects to the left of them, etc. However, postacutely, patients who recover seem to represent space in the same way as healthy subjects, and no experiment till date has shown that recovered patients have any abnormalities in this respect. In such cases, prima facie, it seems rather safe to say that cognitive functions are realizable in different neural substrates at the level of "cognitive domains." So, at this most general level, there seems to be a 1:X relationship between cognitive states and brain states. On this background, it would seem plausible to also suggest that this is true at least at the task- and domain-specific level as well. Some studies, however, can be seen as challenging to this view.

Crosson et al. (2007) developed a method in which aphasic patients were trained in a naming task in a somewhat complex setup designed to achieve activation of right hemisphere mechanisms. Described in a simplified manner, the patients were required to perform a complex task using their left (right hemisphere-associated) hand as the initial step of the naming task. Theoretically, this voluntary activity can achieve a right hemisphere activation, which might facilitate other - more specifically language-associated activations within that side of the brain. Although not globally effective, the method appeared to produce a significant level of success in many aphasic patients (Crosson et al., 2007). In a subsequent study Crosson et al. (2009) investigated - by the use of fMRI - the neural activation patterns associated with the improved performance of the naming task under such circumstances. The 
results showed that the naming activity per se (not the performance of the complex motor task) was associated with an increase in focal activation within parts of the right frontal lobe. Apparently, this activation was associated with the linguistic improvements - in one or another respect representing a rightward "shift" of language processing. It is beyond doubts that such achievements are clinically significant and may constitute an important avenue in future developments of therapeutic interventions. What remains open to question, however, is the issue of exactly what is mediated by the frontal regions activated within the right hemisphere.

In most clinical studies, it is extremely hard or even impossible to step beyond the demonstration that a particular recovery process is accompanied by a particular focal activation. In animal models, however, there has been a certain level of success in achieving an analysis of whether or not a post-traumatic ability to mediate a given task performance is in reality the same as the normal mediation of that task. A couple of examples will serve to demonstrate that apparently similar task performances upon further scrutiny reveal themselves to be dissimilar.

Lepore et al. (1985) investigated in cats whether information relevant to the performance of a visual discrimination task can be transferred subcallosally (without the presence of the corpus callosum) between the two hemispheres. The cats of the study were subjected to split-brain operations in which the corpus callosum was severed. Initially, the discrimination task was acquired exclusively via one eye (thereby conveying the information to only one hemisphere - since the optic chiasm had been split). Subsequently, the ability to perform the visual discrimination task was tested via the other (untrained) eye - thereby testing the availability of discrimination-associated information to the untrained hemisphere. This test of subcallosal transfer of visual discriminationrelevant information was performed in two separate experimental setups: a classic two-choice discrimination box (a maze-type setup) in which food was offered as a reinforcement and a jumping-stand in which the motivation for successful task performance is the avoidance of an unpleasant fall. While the cats were able to demonstrate a subcallosal transfer of information in the jumping-stand, no such postlesional ability was demonstrated in the two-choice discrimination box.

In another experiment studying rats with lesions within the prefrontal system, it was also found that animals subjected to a focal lesion were in one experimental setup fully able to perform a task normally associated with the injured structure. But when tested in another version of the same task the animals were clearly impaired (Mogensen et al., 1987). Normally, across species an impaired performance of spatial delayed alternation tasks is considered a key symptom of injury within the prefrontal cortex or associated structures such as the prefrontal part of the neostriatum (e.g., Mogensen, 2003, 2011b; Mogensen et al., 2007, 2008). Mogensen et al. (1987) tested rats subjected to lesions of the prefrontal part of the neostriatum (in which even the prefrontal cortex was likely to have been rendered inoperational since the lesion of the neostriatum also destroyed fibers, which are essential to the normal functioning of the prefrontal cortex) in two variants of spatial delayed alternation tasks. One of these tasks was administered in a T-maze while the other was performed in an operant chamber. In the T-maze, two spatially separate positions (in the form of the two arms) are the goals toward which responses are to be made and the animal is removed from the apparatus between trials. In the operant chamber, choices are made between two spatially separate retractable levers situated in one wall of the chamber and the levers are retracted from the chamber between trials. While the animals demonstrated an impairment when tested in the T-maze, the test procedure administered in the operant chamber did not reveal any lesion-associated impairment - in spite of the fact that the operant chamber-based procedures imposed the same demands as the procedures in the T-maze regarding what are believed to be the crucial cognitive parameters such as intertrial delays and the necessity to constantly alternate between spatially dissimilar positions.

These two examples show that although neural mechanisms spared by the lesion in question (lesions of the corpus callosum or the prefrontal region of the neostriatum) are able to successfully mediate certain variants of a particular task, the neural mechanisms within those spared parts of the brain are (at least initially) unable to allow a successful task performance under circumstances which according to the formal task requirements are the same but are realized in another test-environment - thereby calling for at least partly different cognitive and neural mechanisms.

The third and final example from animal model-based studies addresses more specifically the mechanisms of a post-traumatic recovery process. Rats subjected to bilateral removal of the prefrontal cortex, bilateral hippocampal lesions in the form of transections of the fimbria-fornix or a combination of these two lesions were studied in a water maze-based allocentric place learning task of the mapping type by Mogensen et al. (2004). All three brain injured groups demonstrated an impaired task acquisition. While the group subjected to prefrontal lesions in isolation showed a relatively mild impairment, the groups subjected to hippocampal lesions in isolation or the combined lesions of the hippocampus and the prefrontal cortex, respectively, were equally and severely impaired. All animals did, however, eventually recover to a fully normal proficiency of task performance. Neither the speed of recovery nor the parameters of task performance revealed any difference between the two groups subjected to hippocampal lesions - begging the conclusion that if the hippocampus has been injured, the presence or absence of prefrontal mechanisms makes no difference to the mediation of task performance. Such a conclusion did, however, turn out to be a fallacy. Upon completion of the recovery training, all groups of animals were subjected to a series of "challenges" which aimed at demonstrating potential differences in: (1) the neural substrates mediating the successful task performance in the recovered animals, and (2) the cognitive strategies employed by various groups. These challenges demonstrated that when the hippocampus had been injured in isolation, the mediation of the successful recovery of the task received significant contributions from the prefrontal cortex. Obviously, such contributions were absent in the group subjected to lesions of both the hippocampus and the prefrontal cortex, and in these animals the most likely substrate of the successful post-traumatic functional recovery was the parietal association cortex. Furthermore, the two hippocampally lesioned groups differed significantly regarding the cognitive strategies employed (although these strategies were equally successful). In the presence of the prefrontal cortex, hippocampally lesioned animals were able to demonstrate an apparently normal level of cognitive representa- 
tion of the position of the goal position (the platform in the water maze) while such a representation seemed to be absent in animals subjected to the combined lesion.

We would argue that all these three examples, in quite different ways, illustrate that cognitive functions are realizable in different neural substrates at the level of "cognitive domains." However, they all serve to cast doubts on the conclusion that the "second level phenomena" (domain- and task-specific functions) are multiple realized as well. An easy counter-argument to the position that cognition is multiple realized at the most general level only, could be that no matter how many examples one may line up of the lack of such realization, one needs only one certain example of successful multiple realization in order to show that this is possible in principle. Against this, proponents of the position that multiple realization occurs exclusively at the level of "cognitive domains" could argue that in each case where we yet have not found limitations to multiple realizations of cognitive functions at the "second level," the methods used have simply not been sufficiently exhaustive.

In conclusion, we believe it is relatively safe to say that multiple realization occurs at the "cognitive domain" level. At the lowest level of our model, the level of "basic functions," we believe it is too early to say anything specific as research on neuroplasticity is not yet at a sufficiently advanced stage. Currently, the discussion may focus on the "second level functions" where arguments may be formed for either position. If the position is correct that functions at this level are not subject to multiple realization, it would lead to the hypothesis that basic functions are not either.

\section{LEVELS OF ANALYSIS IN CONSCIOUSNESS}

One may now reasonably question whether this approach is specific for neural substrates of cognition, or whether it is applicable to other aspects of mental phenomena, e.g., the study of NCC. As mentioned above, cognitive states are defined with regards to their functions, whereas consciousness is defined as subjective experience (Nagel, 1974). Many leading theories, trying to pinpoint an NCC, claim that certain brain areas are necessary for a person to be conscious of particular contents, e.g., Milner and Goodale (Milner and Goodale, 1995; Goodale, 2007) who argue that ventral projection streams from primary visual cortex are necessary for visual consciousness, and diverse, even more influential theories argue that the prefrontal cortex is necessary (Crick and Koch, 1998; Dehaene and Naccache, 2001). Other theories seem more to focus on specific types of processes than specific brain areas (e.g., Tononi, 2004; Lamme, 2010).

Interestingly, in most cases, studies of NCC's are rather explicitly following a definition introduced by Chalmers (2000) that an NCC should be the minimally neural representational system sufficient for representation of that content in consciousness. This definition is in itself open to multiple realization of consciousness, which leaves it as an empirical question only whether one should think of specific conscious experiences as related to brain processes in a 1:1 or 1:X fashion.

Before proceeding, an important initial difference should however be considered. As we consider consciousness to be identical to subjective experience, which at large is the typical position in NCC literature (Seth et al., 2008), we cannot determine whether two instances of a specific conscious experience are identical based on behavioral measures. Instead, we are fully dependent on introspection and the introspecting subjects' reports (Naccache, 2006; Overgaard and Overgaard, 2010). Whereas several recent papers have argued that the use of introspection per se is not in conflict with the wish to do "proper science" (Piccinini, 2003; Overgaard, 2006), contrary to the leading notion not many decades ago, a specific problem arises in this context.

Even though it is not within the scope of this paper to fully analyze this problem, it is far from trivial how a person may introspectively compare two "instances" of an experiential state. If you are shown two blue colors in sequence, one lighter than the other, you would probably be able to compare the memories of the two perceptions and conclude that they were not identical. However, let us say you were to report whether you experience the exact same stimulus of a blue color in the exact same way as you did, say, a year ago, or before a brain injury that for a period changed your experience of color, it would certainly be a much more difficult task. One reasonable hypothesis about the identification of an experience of, say, a certain color, is its relation to experiences of other colors. In another example, emotions, most people would be willing to say that the feeling of, say, joy has important similarities with the feeling of joy they experienced as children. Were it not so, this specific emotion would in fact not be recognized as a feeling of joy. Nevertheless, whether joy is experienced in the exact same way is difficult to answer for many methodological reasons - not just, although important, because of memory decay. The feeling of joy may have been recognized and identified as “joy” under different premises when one was a child compared to today, which in important ways may depend on changes in how often one entertain this feeling, under which circumstances the feeling occurs, etc. Of course, the same argument could be pursued with many other examples (different types of emotions, memories, perceptions, etc.).

In recent years, there has been beginning progress in the attempt to develop more sophisticated methods to report subjective experiences adequately (e.g., Hurlburt, 2009; Overgaard and Timmermans, 2010; Sandberg et al., 2010), yet the field is far from giving exact methodological solutions to problems as those sketched above. Nevertheless, we believe the field is sufficiently mature to separate different analytical levels of experience, which may help this difficult discussion underway. In Table 2 we provide a tentative framework for levels of analysis relevant to this issue.

A first, over-all distinction can be made following Rosenthal's (1997) concept of "creature consciousness" - whether a person or organism is conscious in any sense. This distinction is of special

Table 2 | Levels of analysis in the relation between conscious and neural events. The number of levels and their labeling are tentative.

\begin{tabular}{ll}
\hline Level of analysis & Description \\
\hline "Creature level" & Whether a person or creature is \\
consciousness & conscious at any level or of any content \\
Phenomenal domain & Modality or "category" of contents, e.g., \\
& visual or auditory perceptions, emotions, etc. \\
Phenomenal content & The conscious experience itself with its contents \\
Character of content & How the content is manifested - with \\
& some degree of clarity, intensity, etc.
\end{tabular}


relevance in the discussion of coma or vegetative state patients where it is still unclear whether we should conceive of them as fully unconscious (yet with some intact cognitive processes) or as conscious to some extent (Owen et al., 2006; Overgaard, 2009). For patients who have lost consciousness for a period and recover it later, one possible interpretation would be that consciousness is now realized in a different neural substrate. This would be a case of NCC plasticity at a most general level.

A further distinction, we believe, can be made between this overall level of analysis and another level - "phenomenal domains," e.g., visual experiences, auditory experiences, emotions, thoughts, etc. "Domains," in this case, refer to experiences for which the difference between them are not just the actual content of the experience, but the "modality" or "kind of presentation."

As already now indicated, this level differs from the actual content, which may or may not include simultaneous differences between modality. In other words, at this level, a difference between, say, the visual experience of two different colors differ just like a visual and auditory experience do.

Finally, each content may itself be different from other instances of the same content. For instance, a conscious perceptual content may be present in different degrees of clarity or intensity. It is a theoretical debate worth having, but not possible in this context, whether two "identical" contents with different intensities as a matter of principle form two different contents, or whether one and the same content may vary in different aspects. In this context, we assume the latter position.

It is even more complex in this area of investigation - compared to the discussions above - to find experiments that can be said to reasonably argue for or against plasticity limited to one level.

With regards to the "creature level," some experiments give reason to believe that the NCC may change after recovery from coma. In a PET study of a single subject, global metabolic function decreased substantially in the vegetative state following $\mathrm{CO}$ intoxication - as would be expected. However, after full recovery, the metabolic level stayed low globally (Laureys, 2005). Although some prefrontal areas did increase metabolism, it seems safe to say that whatever areas or processes may be involved in the NCC, they would be different in the pretraumatic and the post-recovery situation.

In one study, Ptito et al. (2008) applied transcranial magnetic stimulation (TMS) to early blind subjects and blindfolded controls. The blindfolded subjects reported experiencing phosphenes following occipital cortex stimulation, as expected from several previous studies (e.g., Amassian et al., 1998). Some of the blind subjects, nevertheless, reported tactile sensations in the fingers. The representation of those fingers appeared to be somatotopically organized within the visual cortex. Importantly, the number of cortical sites at which tactile sensations could be elicited in the blind was related to the number of hours of "Braille reading" per day. The tactile experiences do show that brain areas that normally would be associated with visual processing are now related to tactile experiences, which indicates cross-domain plasticity.

Cross-domain plasticity in case of individuals deprived of input within one modality is not restricted to "alien source" activations of the visual system of the blind. For instance, Lambertz et al. (2005) reported activation of the auditory cortex due to visual stimulation in deaf subjects.
Intra-modal plasticity related to consciousness is, also, rarely studied, yet some experiments give a few indications of such occurrences. For instance, Ramachandran and Hirstein (1998) studied phantom limb phenomena, where the experience of stroking a missing arm was associated with a factual stroking of the face even though tactile inputs from the arm and face map onto different, adjacent, cortical areas. The case is difficult to interpret as clearly showing plasticity in the neural substrate for phenomenal content, as this would demand an argument that phantom arm sensations are fully identical with sensations in a "normal" arm. Unfortunately, very few studies have investigated the "characters of content" empirically (with few exceptions, e.g., Overgaard et al. (2008)), let alone the possibility for plasticity in their neural substrate.

These examples, though clearly not exhaustive, show that an empirical case could be made in favor of NCC plasticity - from a very general level to at least the level of phenomenal content. Counter-arguments could certainly take many different forms. One very general kind of counter-argument would say that in each of the here mentioned examples, the apparent loss of consciousness and its later recovery (in the Laureys case) or the changed correlation between a specific brain area and conscious experiences associated with it (in the other two cases), what has really changed is not the NCC per se, but rather other circumstances which are necessary for the NCC - yet not themselves parts hereof. Stopping the heart from beating would certainly make a person lose consciousness, yet this does not in itself show that the heart is a "correlate of consciousness." According to the definition by Chalmers (2000), as earlier mentioned, we are looking for the minimally sufficient correlate, not a necessary correlate. Therefore, one could argue, these examples need to show that what has happened is in fact a change in the NCC.

Whereas this debate in the present context stands as unresolved, this, at least, gives us a framework in which this debate may take place and potentially be resolved by empirical means.

\section{CONCLUSION}

Taken together, we believe to have offered a general framework in which a qualified debate about the multiple realization of cognitive and conscious events can take place. As mentioned underway, we wish to underline that the number of levels as well as their exact character in the two "models" are highly tentative and may be subject to revision. Nevertheless, we believe they capture the essence of the kind of structure, we will propose.

With this structure, positions in the debate of multiple realizations are no longer different ways to argue for or against the phenomenon as such, but opens up to more nuanced positions, arguing for plasticity at some levels and against plasticity at other.

We have deliberately said very little about the theoretical consequences of these possible positions, yet this is certainly a question worth pursuing in future work. We do believe that advances in this debate could indicate empirical ways to answer otherwise theoretical questions about the mind-brain relation.

\section{ACKNOWLEDGMENTS}

Morten Overgaard was supported by a Starting Grant from the European Research Council. Jesper Mogensen was supported by a grant from the Danish Research Council. 


\section{REFERENCES}

Amassian, V. E., Cracco, R. Q., Maccabee, P. J., Cracco, J. B., Rudell, A. P., and Eberle, L. (1998). Transcranial magnetic stimulation in the study of the visual pathway. J. Clin. Neurophysiol. 15, 288-304.

Ansaldo, A. I., and Arguin, M. (2003). The recovery from aphasia depends on both the left and right hemispheres: three longitudinal case studies on the dynamics of language function after aphasia. Brain Lang. 87, 177-178.

Ansaldo, A. I., Arguin, M., and Lecours, A. R. (2002). The contribution of the right cerebral hemisphere to the recovery from aphasia: a single longitudinal case study. Brain Lang. 82, 206-222.

Arvidsson, A., Collin, T., Kirik, D., Kokaia, Z., and Lindvall, O. (2002). Neuronal replacement from endogenous precursors in the adult brain after stroke. Nat. Med. 8, 963-970.

Bach-y-Rita, P., Collins, C. C., Saunders, F. A., White, B., and Scadden, L. (1969). Vision substitution by tactile image projection. Nature 221, 963-964.

Bach-y-Rita, P., Kaczmarek, K. A., Tyler, M. E., and Garcia-Lara, J. (1998). Form perception with a 49-point electrotactile stimulus array on the tongue: a technical note. J. Rehabil. Res. Dev. 35, 1-7.

Barlow, T. (1877). On a case of double cerebral hemiplegia, with cerebral symmetrical lesions. Br. Med. J. 2, 103-104.

Baumgaertner, A., Schraknepper, V., and Saur, D. (2005). Differential recovery of aphasia and apraxia of speech in an adolescent after infarction of the left frontal lobe: longitudinal behavioral and fMRI data. Brain Lang. 95, 211-212.

Bechtel, W., and Mundale, J. (1999). Multiple realizability revisited: linking cognitive and neural states. Philos. Sci. 66, 175-207.

Berardi, N., Pizzorusso, T., and Maffei, L. (2004). Extracellular matrix and visual cortical plasticity: freeing the synapse. Neuron 44, 905-908.

Berry, M. (1982). Post-injury myelinbreakdown products inhibit axonal growth: an hypothesis to explain the failure of axonal regeneration in the mammalian central nervous system. Bibl. Anat. 23, 1-11.

Block, N., and Fodor, J. (1972). What psychological states are not. Philos. Rev. 81, 159-181.

Chalmers, D. J. (2000). "What is a neural correlate of consciousness?" in Neural Correlates of Consciousness: Empirical and Conceptual Questions, ed. T. Metzinger (Cambridge, MA: MIT Press), 17-40.

Chen, D. F., Jhaveri, S., and Schneider, G.E. (1995). Intrinsic changes in developing retinal neurons result in regenerative failure of their axons. Proc. Natl. Acad. Sci. U.S.A. 92, 7287-7291.

Chen, J., Magavi, S. S., and Macklis, J. D. (2004). Neurogenesis of corticospinal motor neurons extending spinal projections in adult mice. Proc. Natl. Acad. Sci. U.S.A. 101, 16357-16362.

Corbetta, M., Kincade, M. J., Lewis, C. Snyder, A. Z., and Sapir, A. (2005). Neural basis and recovery of spatial attentional deficits in spatial neglect. Nat. Neurosci. 8, 1424-1425.

Crick, F., and Koch, C. (1998). Consciousness and neuroscience. Cereb. Cortex 8, 97-107.

Crosson, B., McGregor, K., Gopinath, K. S., Conway, T. W., Benjamin, M. Chang, Y.-L., Moore, A. B., Raymer, A. M., Briggs, R. W., Sherod, M. G. Wierenga, C. E., and White, K. D. (2007). Functional MRI of language in aphasia: a review of the literature and the methodological challenges. Neuropsychol. Rev. 17, 157-177.

Crosson, B., Moore, A. B., McGregor, K. M., Chang, Y.-L., Benjamin, M. Gopinath, K., Sherod, M.E., Wierenga, C.E., Peck, K. K., Briggs, R.W., Rothi, L. J. G., and White, K. D. (2009). Regional changes in word-production laterality after a naming treatment designed to produce a rightward shift in frontal activity. Brain Lang. 111, 73-85.

Dehaene, S., and Naccache, L. (2001). Towards a cognitive neuroscience of consciousness. Cognition 79, 1-37.

Del Rio, J. A., and Soriano, E. (2007). Overcoming chondroitin sulphate proteoglycan inhibition of axon growth in the injured brain: lessons from chondroitinase ABC. Curr. Pharm. Des. 13, 2485-2492.

Duffau, H., Denvil, D., and Capelle, L. (2002). Long term reshaping of language, sensory, and motor maps after glioma resection: a new parameter to integrate in the surgical strategy. J. Neurol. Neurosurg. Psychiatr. 72 511-516.

Elbert, T., Pantev, C., Weinbruch, C., Rockstroh, B., and Taub, E. (1995) Increased cortical representation of the fingers of the left hand in string players. Science 270, 305-307.

Fawcett, J. W., Housden, E., SmithThomas, L., and Meyer, R. L. (1989). The growth of axons in three-dimensional astrocyte cultures. Dev. Biol. $135,449-458$.

Finger, S., Buckner, R. L., and Buckingham, H. (2003). Does the right hemisphere take over after damage to Broca's area? The Barlow case of 1877 and its history. Brain Lang. 85, 385-395.

Finger, S., and Stein, D. G. (1982). "Vicariation theory and radical reorganization of function," in Brain Damage and Recovery: Research and Clinical Perspectives, eds S. Finger and D. G. Stein (New York: Academic Press), 287-302.

Gizewski, E. R., Gasser, T., de Greiff, A. Boehm, A., and Forsting, M. (2003). Cross-modal plasticity for sensory and motor activation patterns in blind subjects. Neuroimage 19, 968-975.

Goldberg, J. L., Klassen, M. P., Hua, Y., and Barres, B. A. (2002). Amacrinesignaled loss of intrinsic axon growth ability by retinal ganglion cells. Science 296, 1860-1864.

Goodale, M. (2007). "Duplex vision: separate cortical pathways for conscious perception and the control of action," in The Blackwell companion to conscious ness, eds M. Velmans and S. Schneide (Oxford: Blackwell), 616-627.

Hurlburt, R. T. (2009). "Descriptive experience sampling," in Oxford Companion to Consciousness, eds T. Bayne, A. Cleeremanns, and P. Wilken (Oxford: Oxford University Press), 225-227.

Irvine, D. R. F. (2007). Auditory cortical plasticity: does it provide evidence for cognitive processing in the auditory cortex? Hear. Res. 229, 158-170.

Kaczmarek, K. A., Webster, J. G., Bach-yRita, P., and Tompkins, W. J. (1991) Electrotactile and vibrotactile displays for sensory substitutionsystems. IEEE Trans. Biomed. Eng. 38, 1-16.

Karl, A., Birbaumer, N., Lutzenberger W., Cohen, L. G., and Flor, H. (2001) Reorganization of motor and somatosensory cortex in upper extremity amputees with phantom limb pain. $J$ Neurosci. 15, 3609-3618.

Keller-Peck, C. R., Walsh, M. K., Gan, W.-B., Feng, G., Sanes, J. R., and Lichtman, J.W. (2001). Asynchronous synapse elimination in neonatal motor units: studies using GFP transgenic mice. Neuron 31, 381-394.

Laganaro, M., Morand, S., Schwitter, V. Zimmermann, C., and Schnider, A (2008). Normalisation and increase of abnormal ERP patterns accompany recovery from aphasia in the post-acute stage. Neuropsychologia 46, 2265-2273.

Lambertz, N., Gizewski, E. R., de Greiff,A., and Forsting, M. (2005). Cross-moda plasticity in deaf subjects dependent on the extent of hearing loss. Cogn Brain Res. 25, 884-890.

Lamme, V. (2010). How neuroscience will change our view on consciousness. Cogn. Neurosci. 1, 204-220.

Laureys, S. (2005). The neural correlate of (un)awareness: lessons from the vegetative state. Trends Cogn. Sci. (Regul. Ed.) 9, 556-559.

Leavitt, B. R., Hernit-Grant, C. S., and Macklis, J. D. (1999). Mature astrocytes transform into transitional radia glia within adult mouse neocortex that supports directed migration of transplanted immature neurons. Exp. Neurol. 157, 43-57.

Lee, W. C., Huang, H., Feng, G., Sanes, J. R., Brown, E. N., So, P. T., and Nedivi, E. (2006). Dynamic remodeling of dendritic arbors in GABAergic interneurons of adult visual cortex. PLoS Biol.4, e29. doi: 10.1371/journal.pbio.0040029

Lee, W.-C. A., Chen, J. L., Huang, H. Leslie, J. H., Amitai, Y., So, P. T., and Nedivi, E. (2008). A dynamic zone defines interneuron remodeling in the adult neocortex. Proc. Natl. Acad. Sci. U.S.A. 105, 19968-19973.

Lepore, F., Ptito, M., Provencal, C., Bedard, S., and Guillemot, J.-P. (1985). Interhemispheric transfer of visual training in the split-brain cat: effects of the experimental set-up. Can. J. Psychol. 37, 527.

Magavi, S. S., Leavitt, B. R., and Macklis, J. D. (2000). Induction of neurogenesis in the neocortex of adult mice. Nature 405, 951-955

Marsh, E. B., and Hillis, A. E. (2006). Recovery from aphasia following brain injury: the role of reorganization. Prog. Brain Res. 157, 143-156.

McGee, A. W., Yang, Y., Fischer, Q. S., Daw, N. W., and Strittmatter, S. M. (2005). Experience-driven plasticity of visual cortex limited by myelin and Nogo receptor. Science 309, 2222-2226.

Meinzer, M., Flaisch, T., Breitenstein, C., Wienbruch, C., Elbert, T., and Rockstroh, B. (2008). Functional rerecruitment of dysfunctional brain areas predicts language recovery in chronic aphasia. Neuroimage 39, 2038-2046.

Meinzer, M., Obleser, J., Flaisch, T., Eulitz, C., and Rockstroh, B. (2007) Recovery from aphasia as a function of language therapy in an early bilingual patient demonstrated by fMRI. Neuropsychologia 45, 1247-1256.

Merzenich, M. M., and Jenkins, W. M. (1993). Reorganization of cortical representations of the hand following alterations of skin inputs induced by nerve injury, skin island transfers, and experience. J. Hand Ther. 6, 89-104.

Milner, A. D., and Goodale, M. A. (1995). The Visual Brain in Action. Oxford: Oxford University Press.

Mogensen, J. (2003). “Animal models in neuroscience," in Handbook of Laboratory Animal Science, 2nd Edn, Vol. II, Animal Models, eds J. Hau and G. L. van Hoosier (Boca Raton, FL: CRC Press LLC), 95-109.

Mogensen, J. (2011a). Almost unlimited potentials of a limited neural plasticity: levels of plasticity in development and reorganization of the injured brain. J. Conscious. Stud. (in press).

Mogensen, J. (2011b). "Animal models in neuroscience," in Handbook of Laboratory Animal Science, 3rd Edn, 
Vol. II, Animal Models, eds J. Hau and S. Schapiro (Boca Raton, FL: CRC Press LLC), (in press).

Mogensen, J. (2011c). Reorganization in the injured brain: implications for studies of the neural substrate of cognition. Front. Psychol. 2:7. doi: 10.3389/fpsyg.2011.00007

Mogensen, J., Boyd, M. H., Nielsen, M. D., Kristensen, R. S., and Malá, H. (2008). Erythropoietin improves spatial delayed alternation in a T-maze in rats subjected to ablation of the prefrontal cortex. Brain Res. Bull. 77, 1-7.

Mogensen, J., Hjortkjær, J., Ibervang, K. L., Stedal, K., and Malá, H. (2007). Prefrontal cortex and hippocampus in posttraumatic functional recovery: spatial delayed alternation by rats subjected to transection of the fimbriafornix and/or ablation of the prefrontal cortex. Brain Res. Bull. 73, 86-95.

Mogensen, J., Iversen, I. H., and Divac, I. (1987). Neostriatal lesions impaired rats' delayed alternation performance in a T-maze but not in a two-key operant chamber. Acta Neurobiol. Exp. (Wars) 47, 45-54.

Mogensen, J., Lauritsen, K. T., Elvertorp, S., Hasman, A., Moustgaard, A., and Wörtwein, G. (2004). Place learning and object recognition by rats subjected to transection of the fimbria-fornix and/or ablation of the prefrontal cortex. Brain Res. Bull. 63, 217-236.

Mogensen, J., and Malá, H. (2009). Posttraumatic functional recovery and reorganization in animal models. A theoretical and methodological challenge. Scand. J. Psychol. 50, 561-573.

Münte, T. F., Altenmüller, E., and Jäncke, L. (2002). The musician's brain as a model of neuroplasticity. Nat. Rev. Neurosci. 3, 473-478.

Naccache, L. (2006). Is she conscious? Science 313, 1395-1396.

Nagel, T. (1974). What is it like to be a bat? Philos. Rev. 83, 435-450.

Nakatomi, H., Kuriu, T., Okabe, S., Yamamoto, S.-C., Hatano, O., Kawahara, N., Tamura, A., Kirino, T., and Nakafuku, M. (2002). Regeneration of hippocampal pyramidal neurons after ischemic brain injury by recruitment of endogenous neural progenitors. Cell 110, 429-441.

Overgaard, M. (2006). Introspection in science. Conscious. Cogn. 15, 629-633.

Overgaard, M. (2009). How can we know if patients in coma, vegetative state or minimally conscious state are conscious. Prog. Brain Res. 177, 11-19.

Overgaard, M., Fehl, K., Mouridsen, K., and Cleeremans, A. (2008). Seeing without seeing? Degraded conscious vision in a blindsight patient. PLoS ONE 3, e3028. doi: 10.1371/journal. pone. 0003028
Overgaard, M., and Overgaard, R. (2010). Neural correlates of contents and levels of consciousness. Front. Psychol. 1:164. doi: 10.3389/ fpsyg.2010.00164

Overgaard, M., and Timmermans, B. (2010). "How unconscious is subliminal perception?" in Handbook of Phenomenology and Cognitive Science, eds D. Schmicking and S. Gallagher (Heidelberg: SpringerVerlag), 501-519.

Owen, A. M., Coleman, M. R., Boly, M., Davis, M. H., Laureys, S., and Pickard, J.D. (2006). Detecting awareness in the vegetative state. Science 313, 1402

Perani, D., Cappa, S. F., Tettamanti, M., Rosa, M., Scifo, P., Miozzo, A., Basso, A., and Fazio, F. (2003). A fMRI study of word retrieval in aphasia. Brain Lang. 85, 357-368.

Piccinini, G. (2003). Data from introspective reports: upgrading from commonsense to science. J. Conscious. Stud. 10, 141-156.

Pizzorusso, T., Medini, P., Berardi, N., Chierzi, S., Fawcett, J.W., and Maffei, L. (2002). Reactivation of ocular dominance plasticity in the adult visual cortex. Science 298, 1248-1251.

Ptito, M., Fumal, A., de Noordhout, A. M., Schoenen, J., Gjedde, A., and Kupers, R. (2008). TMS of the occipital cortex induces tactile sensations in the fingers of blind Braille readers. Exp. Brain Res. 184, 193-200.

Ptito, M., Moesgaard, S. M., Gjedde, A., and Kupers, R. (2005). Cross-modal plasticity revealed by electrotactile stimulation of the tongue in the congenitally blind. Brain 128, 606-614.

Rakic, P. (1971). Guidance of neurons migrating to the fetal monkey neocortex. Brain Res. 33, 471-476.

Rakic, P. (1985). "Mechanisms of neuronal migration in developing cerebellar cortex," in Molecular Basis of Neural Development, eds G. M. Edelman, W. M. Cowan, and E. Gull (New York: Wiley), 139-160.

Ramachandran, V. S., and Hirstein, W. (1998). The perception of phantom limbs. Brain 121, 1603-1630.

Recanzone, G. H., Schreiner, C. E., and Merzenich, M. M. (1993). Plasticity in the frequency representation of primary auditory cortex following discrimination training in adult owl monkeys. J. Neurosci. 13, 87-103.

Robertson, D., and Irvine, D. R. F. (1989). Plasticity of frequency organization in auditory cortex of guinea pigs with partial unilateral deafness. J. Comp. Neurol. 282, 456-471.

Rosenthal, D. (1997). "A theory of consciousness," in The Nature of Consciousness, eds N. Block, O. Flanagan, and G. Güzeldere (Cambridge, MA: MIT Press), 729-753.
Sandberg, K., Timmermans, B., Overgaard, M., and Cleeremans, A. (2010). Measuring consciousness: is one measure better than the other? Conscious. Cogn. 19, 1069-1078.

Schäfer, R., Dehn, D., Burbach, G. J., and Deller, T. (2008). Differential regulation of chondroitin sulfate proteoglycan mRNAs in the denervated rat fascia dentata after unilateral entorhinal cortex lesion. Neurosci. Lett. 439, 61-69.

Scharff, C., Kirn, J. R., Grossman, M., Macklis, J. D., and Nottebohm, G. (2000). Targeted neuronal death affects neuronal replacement and vocal behavior in adult songbirds. Neuron 25, 481-492.

Scheich, H. (1991). Auditory cortex: comparative aspects of maps and plasticity. Curr. Opin. Neurobiol. 1, 236-247.

Schwab, M. E., and Thoenen, H. (1985). Dissociated neurons regenerate into sciatic but not optic nerve explants in culture irrespective of neurotrophic factors. J. Neurosci. 5, 2415-2423.

Seth, A, Dienes, Z, Cleeremans, A, Overgaard, M., and Pessoa, L. (2008). Measuring consciousness: relating behavioural and neurophysiological approaches. Trends Cogn. Sci. (Regul. Ed.) 12, 314-321.

Shapiro, L. (2004). The Mind Incarnate. Cambridge, MA: MIT Press.

Shapiro, L. (2008). How to test for multiple realization. Philos. Sci. 75, 514-525.

Slavin, M. D., Laurence, S., and Stein, D. G. (1988). "Another look at vicariation," in Brain Injury and Recovery: Theoretical and Controversial Issues, eds S. Finger, T. E. LeVere, C. R. Almli, and D. G. Stein (New York: Plenum Press), 165-178.

Specht, K., Zahn, R., Willmes, K., Weis, S., Holtel, C., Krause, B. J., Herzog, H., and Huber, W. (2009). Joint independent component analysis of structural and functional images reveals complex patterns of functional reorganisation in stroke aphasia. Neuroimage 47 2057-2063.

Szaflarski, J. P., Eaton, K., Ball, A. L., Banks, C., Vannest, J., Allendorfer, J. B., Page, S., and Holland, S. K. (2010). Poststroke aphasia recovery assessed with functional magnetic resonance imaging and a picture identification task. J. Stroke Cerebrovasc. Dis. 1-10.

Thai-Van, H., Micheyl, C., Norena, A., Veuillet, E., Gabriel, D., and Collet, L. (2007). Enhanced frequency discrimination in hearing-impaired individuals: a review of perceptual correlates of central neural plasticity induced by cochlear damage. Hear. Res. 233 , 14-22.

Thomas, C., Altenmüller, E., Marckmann, G., Kahrs, J., and Dichgans, J. (1997). Language processing in aphasia: changes in lateralization patterns during recovery reflect cerebral plasticity in adults. Electroencephalogr. Clin. Neurophysiol. 102, 86-97.

Thompson, C. K., den Ouden, D.-B., Bonakdarpour, B., Garibaldi, K., and Parrish, T. B. (2010). Neural plasticity and treatment-induced recovery of sentence processing in agrammatism. Neuropsychologia 48, 3211-3227.

Thulborn, K. R., Carpenter, P. A., and Just, M. A. (1999). Plasticity of languagerelated brain function during recovery from stroke. Stroke 30, 749-754.

Tononi, G. (2004). An information integration theory of consciousness. BMC Neurosci. 5, 42. doi: 10.1186/1471-2202-5-42

Weiss, T., Miltner, W. H. R., Huonker, R., Friedel, R., Schmidt, I., and Taub, E. (2000). Rapid functional plasticity of the somatosensory cortex after finger amputation. Exp. Brain Res. 134, 199-203.

Wilms, I., and Mogensen, J. (in press). Dissimilar outcomes of apparently similar procedures as a challenge to clinical neurorehabilitation and basic research - when the same is not the same. Neurorehabilitation.

Xerri, C., Coq, J., Merzenich, M., and Jenkins, W. (1996). Experienceinduced plasticity of cutaneous maps in the primary somatosensory cortex of adult monkeys and rats. J. Physiol. 90, 277-287.

Yang, T. T., Gallen, C. C., Ramachandran, V. S., Cobb, S., Schwartz, B. J., and Bloom, F. E. (1994). Noninvasive detection of cerebral plasticity in adult human somatosensory cortex. Neuroreport 5, 701-704.

Conflict of Interest Statement: The authors declare that the research was conducted in the absence of any commercial or financial relationships that could be construed as a potential conflict of interest.

Received: 04 October 2010; paper pending published: 14 December 2010; accepted: 13 April 2011; published online: 27 April 2011. Citation: Overgaard M and Mogensen J (2011) A framework for the study of multiple realizations: the importance of levels of analysis. Front. Psychology 2:79. doi: 10.3389/fpsyg.2011.00079

This article was submitted to Frontiers in Consciousness Research, a specialty of Frontiers in Psychology.

Copyright (c) 2011 Overgaard and Mogensen. This is an open-access article subject to a non-exclusive license between the authors and Frontiers Media SA, which permits use, distribution and reproduction in other forums, provided the original authors and source are credited and other Frontiers conditions are complied with. 